\title{
Perception About Transplant of Rural and Urban Patients With Chronic Kidney Disease; A Qualitative Study
}

\author{
Nasrollah Ghahramani ${ }^{1, *}$; Chloe Wang ${ }^{2}$; Ali Sanati-Mehrizy ${ }^{2}$; Ankita Tandon ${ }^{1}$ \\ ${ }^{1}$ Department of Medicine, Pennsylvania State University College of Medicine, Hershey, USA \\ ${ }^{2}$ Pennsylvania State University College of Medicine, Hershey, USA \\ ${ }^{*}$ Corresponding author: Nasrollah Ghahramani, Department of Medicine, Pennsylvania State University College of Medicine, Hershey, USA. Tel: +1-7175318156, Fax: +1-7175316776, E- \\ mail: nghahramani@hmc.psu.edu
}

Received: October 28, 2013; Revised: November 5, 2013; Accepted: January 27, 2014

Background: Chronic kidney disease (CKD) is a worldwide public health problem with increasing incidence and prevalence and associated expenses.

Objectives:To explore different perceptions of rural and urban patients with chronic kidney disease (CKD) about kidney transplant. Patients and Methods: We conducted four focus groups, each including 5 or 6 patients with stage 5 CKD or end stage renal disease living in a rural or urban area. Open-ended questions probed patient familiarity with kidney transplant, perceptions of benefits of kidney transplant, perceived barriers to kidney transplant, and views about living donation. All the sessions were recorded and professionally transcribed. Responses were pooled, de-identified, and analyzed using qualitative thematic content analysis.

Results: Urban patients were more likely to receive supplementary information and being strongly encouraged by their nephrologists to seek transplant. All participants acknowledged "independence" as the main advantage of transplantation. Increased freedom to travel and improved life expectancy were mentioned only among the urban groups. The main themes in all groups regarding perceived barriers to transplant were the tedious pre-transplant testing and workup expenses. Among rural groups, there was a perception that distance from transplant centers impedes transplant evaluation. Religious reasons favoring and opposing transplant were mentioned by participants in a rural group. Some members contended that since illness is God's will, we should not change it. Others in the same group argued that "God is not ready for us to give up". Praise and gratitude for the living donor were expressed in all groups, but concerns about donor's outcome were discussed only within the rural groups. In discussing preference about known or anonymous donors, members of an urban group mentioned favoring an anonymous donor, citing unease with a sense of life-long indebtedness.

Conclusions: Observed differences in perceptions among rural and urban patients about aspects of transplant may contribute to geographic disparities in transplant. The findings could be helpful to guide future individualized, culturally sensitive educational interventions about transplant for patients with CKD.

Keywords:Focus Groups; Geography; Disparity

\section{Background}

Chronic kidney disease (CKD) is a worldwide public health problem with increasing incidence and prevalence and associated expenses $(1,2)$. The most accepted treatment for end stage renal disease (ESRD) is in-center hemodialysis worldwide $(3,4)$. Transplant leads to improved survival, a better quality of life, and lower costs compared to long-term dialysis. It is the treatment of choice for most patients $(5,6)$. Among transplant options, pre-emptive living donor transplant is associated with better graft and patient survival (7). Despite well-known advantages of renal transplant, there is a wide geographic variation in the rate of kidney transplant $(8,9)$. Patients living in rural regions are up to $15 \%$ less likely to be placed on the waiting list (10). The contribution of patient attitudes and perceptions to geographic disparities in transplant rates is not well defined. We hypothesize that urban-rural differences in transplant rates reflect differences in perceptions of patients about transplant.

\section{Objectives}

Our aim was to explore rural-urban differences in perception about kidney transplant among patients with CKD. Specifically, we investigated four themes, which included familiarity with transplant, perceptions of benefits of transplant, perceived barriers to kidney transplant, and views about living donation among patients with CKD.

\section{Patients and Methods}

\subsection{Study Design and Recruitment of Participants}

The Institutional Review Board of Penn State College of

Implication for health policy/practice/research/medical education:

This study improves our understanding of the general areas of concern about transplant among rural and urban patients with kidney disease. The findings from this qualitative study facilitate developing quantitative survey studies, which would target the issue of disparities in transplantation.

Copyright (C) 2014, Nephrology and Urology Research Center; Published by Kowsar Corp. This is an open-access article distributed under the terms of the Creative Commons Attribution License, which permits unrestricted use, distribution, and reproduction in any medium, provided the original work is properly cited. 
Medicine approved the research protocol. The protocols conformed to the guidelines of the 1975 Helsinki Declaration. Written consent forms were completed by each subject. Four 2-hour focus group sessions were planned to have two focus group sessions each for patients living in urban and rural areas, respectively. Eligible participants were adult patients with stage five CKD or ESRD, willing to participate in group discussion and physically able to attend focus group meetings. We recruited participants by posting flyers in dialysis units and nephrologists' offices in the central region of Pennsylvania. The flyers described the purpose of the study, the intended duration, date, time, and location of the sessions, and the honoraria provided for participation. Those interested were asked to directly contact the principal investigator to obtain further details. We recruited 10 participants for each focus group to ensure a group of at least five participants. Participants were assigned to one of four focus groups depending on their home address either a rural or an urban area, based on rural-urban commuting area codes, and whether they preferred a morning or an afternoon meeting. The principal investigator contacted each volunteer by telephone one day before the group meeting to confirm his or her participation.

\subsection{Topic Guide}

We developed a topic guide based on literature review and peer discussion. The guide was revised following pilot testing among seven dialysis patients at the principal investigator's institution. Key questions consisted of broadly stated open-ended queries probing the participants' familiarity with transplant, perceptions of benefits of transplant, perceived barriers to transplant, and views about living donation.

\subsection{Data Collection}

The principal investigator initiated each session by introducing himself and asking participants to introduce themselves, with the option of using an alias. He then described the research and answered questions. The participants were reassured of the confidentiality of their comments and were also reassured that all collected information, including audio-recordings of the sessions would be anonymous and eliminated after completion of data analysis. The participants were informed about their right to withdraw from the study at any point. Each participant then completed the informed consent, including consent to audio recording and transcription of the discussion and completed an anonymous demographic questionnaire. For consistency, the principal investigator moderated all sessions using reflective probes for clarification of statements. To ensure balanced participation and to avoid domination by any group member, we actively encouraged all participants to express their views (11). This approach minimizes the potential for ac- quiescence bias, in which the group members tend to conform to the dominant view (12). All sessions were audio recorded and transcribed verbatim by a professional transcriptionist.

\subsection{Data Analysis}

Quality review of each session's transcript was conducted. Two reviewers independently reviewed each transcript to identify recurrent response themes. Either the group or the individual may be considered for data analysis of qualitative studies (13). In our study, we deidentified pooled responses from individuals within each category of respondents (urban and rural). We then used qualitative thematic content analysis $(14,15)$, by thoroughly searching the transcripts to identify dominant and recurrent themes.

\section{Results}

Four separate focus groups were conducted with 23 participants. Each focus group consisted of five or six patients with CKD living in rural $(n=11)$ or urban area $(n=12)$. Demographic characteristics of the participants are outlined in Table 1 . The dominant themes and subthemes emerged during focus group discussions of related topics are outlined below and in Table 2. Urban participants were more likely to have received supplementary information, such as videos or pamphlets, from their nephrologists about transplant. Urban participants were also more likely to being strongly encouraged by their nephrologists to seek transplant. Both urban and rural participants acknowledged "more independence" as the main advantage of kidney transplant compared with dialysis. "Increased freedom to travel" and "improved life expectancy" were mentioned only among the urban participants as advantages. When exploring some of the perceived barriers about transplant, both urban and rural groups noted the tedious

\begin{tabular}{|c|c|c|c|c|}
\hline & \multicolumn{2}{|c|}{ Rural } & \multicolumn{2}{|c|}{ Urban } \\
\hline & $\begin{array}{l}\text { Group } 1 \\
(\mathbf{n}=6)\end{array}$ & $\begin{array}{c}\text { Group } 2 \\
(\mathbf{n}=5)\end{array}$ & $\begin{array}{c}\text { Group } 3 \\
(n=6)\end{array}$ & $\begin{array}{c}\text { Group } 4 \\
(n=6)\end{array}$ \\
\hline Female & 2 & 3 & 3 & 3 \\
\hline Caucasian & 5 & 4 & 3 & 5 \\
\hline Some college & 1 & 1 & 3 & 1 \\
\hline On dialysis & 5 & 3 & 5 & 4 \\
\hline $\begin{array}{l}\text { Referred for Trans- } \\
\text { plant }\end{array}$ & 3 & 2 & 3 & 2 \\
\hline Listed for transplant & 2 & 1 & 2 & 2 \\
\hline \multirow[t]{2}{*}{ Age, Mean \pm SD } & $61 \pm 15.6$ & $65 \pm 9.5$ & $65 \pm 9.9$ & $59 \pm 16.5$ \\
\hline & \multicolumn{2}{|c|}{$63 \pm 12.1$} & \multicolumn{2}{|c|}{$62 \pm 13.1$} \\
\hline
\end{tabular}

\footnotetext{
a No significant difference.
} 
Ghahramani N et al.

\begin{tabular}{|c|c|c|}
\hline \multirow[t]{2}{*}{ Themes } & \multicolumn{2}{|c|}{ Sub-themes } \\
\hline & Rural & Urban \\
\hline Familiarity with KT & & received supplemental info (video, pamphlet) \\
\hline Encouraged by nephrologists to pursue KT & & strongly encouraged \\
\hline \multirow[t]{3}{*}{ Main advantage of KT vs. dialysis } & independence & independence \\
\hline & & freedom to travel \\
\hline & & improved life expectancy \\
\hline Perceived barriers to KT & tedious testing process and cost & tedious testing process and cost \\
\hline \multicolumn{3}{|c|}{ distance from center } \\
\hline \multicolumn{3}{|c|}{ religious reasons opposing KT } \\
\hline \multirow[t]{2}{*}{ Perceptions about living donation } & praise and gratitude for the donor & praise and gratitude for the donor \\
\hline & concern about donor outcome & $\begin{array}{l}\text { prefer anonymous donor (unease with sense } \\
\text { of life-long indebtedness to known donor) }\end{array}$ \\
\hline
\end{tabular}

a Abbreviation: KT, kidney transplant.

pre-transplant testing processes and workup expenses. Rural groups also noted the distance from transplant center as an additional impediment to transplant evaluation. Religious reasons favoring or opposing transplant were mentioned by participants in one of the rural focus groups. Some members contended that since "illness is God's will, we should not change it". Other members in the same group argued, "God is not ready for us to give up". Praise and gratitude for the living donor were expressed in all groups; but concerns about donor's outcome were discussed only within the two rural groups. In discussing preference about known or anonymous donors, members of one of the urban focus groups mentioned that they would prefer an anonymous donor, citing the unease with a sense of life-long indebtedness to the organ donor.

\section{Discussion}

This qualitative study yielded valuable insight into some of the factors affecting patient decisions about transplant. Comparing urban and rural patients with CKD, we found that both groups considered independence as the main advantage of kidney transplant, and tedious testing and cost as the major barrier to transplant and praise and gratitude for the donor. We also identified differences in perceptions of rural and urban patients about advantages of kidney transplant, barriers to transplant and views about living donation. Urban patients provided more details about the advantage of kidney transplant compared with dialysis, citing freedom to travel and improved life expectancy. This finding is consistent with the discussion among urban patients who received supplemental information about transplant and encouragement from their nephrologists to pursue transplant. It is not surprising that rural patients were more likely to mention distance from the transplant center as a major barrier to transplant.

The fact that concern about donor outcome was discussed in both rural groups but in none of the urban groups was interesting. It was also interesting that urban patients, given the option, noted preference for anonymous donor to someone known to them, expressing unease with the sense of life-long indebtedness. These two findings are congruent with suggestions and findings in the social sciences literature that life in rural areas is characterized by values that emphasize reciprocity and prioritize social obligation and duty. Adaptation to urban life, on the other hand, is associated with progressive individualism and self-prioritization (16-18). Religion influence on views about transplant was mentioned only among one of the rural groups. The role of religion in decision making related to health care among the rural population has been previously described (19). The main limitation of our study was selection bias. It is likely that, compared with other regions and countries, patients from central Pennsylvania have different views and perceptions about transplant. However, the principle conclusion of the study is the need to consider diverse perceptions and concerns about transplant, based on geographic regions. Concern about the notes and audio-taping may have potentially led to reluctance to participate. To overcome this reluctance, the moderator reviewed the purpose of the notes and recordings and explained procedures for protecting and handling confidential information to reassure the participants of confidentiality. Another potential limitation of our study was the moderator bias. The personal views and biases of the moderator, a transplant nephrologist, might have dominated the course of discussion (20). To minimize the 
moderator bias, we conducted semi-structured sessions, by using open-ended questions, which had been modified by pilot-testing.

We conclude that physicians should be aware of beliefs, concerns and fears of patients in discussing the option of transplant. The themes identified in this qualitative study would facilitate the development of quantitative studies of geographic variations in patient perceptions about transplant. The findings could be helpful to guide future individualized, culturally sensitive educational interventions about transplant for patients with CKD.

\section{Acknowledgements}

Dr. Ghahramani is grateful to Dr. Roger Anderson, Dr. Kristen Kjerulff, and Dr. Eugene Lengerich for their mentorship and valuable advice. The authors wish to thank the individuals who participated in the pilot phase and the focus group discussions.

\section{Authors' Contribution}

All authors contributed significantly to the design, analysis, and interpretation of data, as well as drafting the manuscript and providing significant intellectual content. All of the authors approved this manuscript for publication.

\section{Financial Disclosure}

The authors have no conflict of interest.

\section{Funding/Support}

Dr. Nasrollah Ghahramani is supported by award number K23DK084300 from the National Institute of Diabetes and Digestive and Kidney Diseases. The content is solely the responsibility of the authors and does not necessarily represent the official views of the National Institute of Diabetes and Digestive and Kidney Diseases or the National Institutes of Health.

\section{References}

1. Eknoyan G, Lameire N, Barsoum R, Eckardt KU, Levin A, Levin N, et al. The burden of kidney disease: improving global outcomes. Kidney Int. 2004;66(4):1310-4

2. Lameire N, Eknoyan G, Barsoum R, Eckardt KU, Levin A, Levin N, et al. A new initiative in nephrology: 'Kidney disease: improving global outcomes'. Contrib Nephrol. 2005;149:90-9.

3. Collins AJ, Foley RN, Chavers B, Gilbertson D, Herzog C, Johansen K, et al. 'United States Renal Data System 2011 Annual Data Report: Atlas of chronic kidney disease \& end-stage renal disease in the United States. Am J Kidney Dis. 2012;59(1 Suppl 1):A7.

4. van de Luijtgaarden MW, Jager KJ, Stel VS, Kramer A, Cusumano A, Elliott RF, et al. Global differences in dialysis modality mix: the role of patient characteristics, macroeconomics and renal service indicators. Nephrol Dial Transplant. 2013;28(5):1264-75.

5. Abecassis M, Bartlett ST, Collins AJ, Davis CL, Delmonico FL, Friedewald JJ, et al. Kidney transplantation as primary therapy for end-stage renal disease: a National Kidney Foundation/Kidney Disease Outcomes Quality Initiative (NKF/KDOQITM) conference. Clin J Am Soc Nephrol. 2008;3(2):471-80.

6. Wolfe RA, Ashby VB, Milford EL, Ojo AO, Ettenger RE, Agodoa LY, et al. Comparison of mortality in all patients on dialysis, patients on dialysis awaiting transplantation, and recipients of a first cadaveric transplant. N Engl J Med. 1999;341(23):1725-30.

7. Milton CA, Russ GR, McDonald SP. Pre-emptive renal transplantation from living donors in Australia: effect on allograft and patient survival. Nephrology (Carlton). 2008;13(6):535-40.

8. Castledine CI, Gilg JA, Rogers C, Ben-Shlomo Y, Caskey FJ. How much of the regional variation in RRT incidence rates within the UK is explained by the health needs of the general population? Nephrol Dial Transplant. 2012;27(10):3943-50.

9. Hommel K, Rasmussen S, Kamper AL, Madsen M. Regional and social inequalities in chronic renal replacement therapy in Denmark. Nephrol Dial Transplant. 2010;25(8):2624-32.

10. Axelrod DA, Guidinger MK, Finlayson S, Schaubel DE, Goodman DC, Chobanian M, et al. Rates of solid-organ wait-listing, transplantation, and survival among residents of rural and urban areas. JAMA. 2008;299(2):202-7.

11. Nyamathi A, Shuler P. Focus group interview: a research technique for informed nursing practice. J Adv Nurs. 1990;15(11):1281-8.

12. Crawford M, Acorn S. Focus groups: their use in administrative research. J Nurs Adm. 1997;27(5):15-8.

13. Kidd PS, Parshall MB. Getting the focus and the group: enhancing analytical rigor in focus group research. Qual Health Res. 2000;10(3):293-308.

14. Graneheim UH, Lundman B. Qualitative content analysis in nursing research: concepts, procedures and measures to achieve trustworthiness. Nurse Educ Today. 2004;24(2):105-12.

15. Burnard P.A method of analysing interview transcripts in qualitative research. Nurse Educ Today. 1991;11(6):461-6.

16. Phillips CD, McLeroy KR. Health in rural America: remembering the importance of place. Am J Public Health. 2004;94(10):1661-3.

17. Greenfield PM. The changing psychology of culture from 1800 through 2000. Psychol Sci. 2013;24(9):1722-31.

18. Inglehart R, Baker WE. Modernization, cultural change, and the persistence of traditional values. Am Soci Rev. 2000:19-51.

19. Behringer B, Krishnan K. Understanding the role of religion in cancer care in Appalachia. South Med J. 2011;104(4):295-6.

20. Morgan C, Park E, Cortes DE. Beliefs, knowledge, and behavior about cancer among urban Hispanic women. J Natl Cancer Inst Monogr. 1995(18):57-63. 